\title{
A rigorous analysis using optimal transport theory for a two-reflector design problem with a point source
}

\author{
Tilmann Glimm \\ Department of Mathematics \\ Western Washington University, Bellingham, WA 98225
}

August 17, 2009

2000 Mathematics Subject classification: 78A05, 49J20; Keywords: reflector design, geometric optics, optimal transportation

\begin{abstract}
We consider the following geometric optics problem: Construct a system of two reflectors which transforms a spherical wavefront generated by a point source into a beam of parallel rays. This beam has a prescribed intensity distribution. We give a rigorous analysis of this problem. The reflectors we construct are (parts of) the boundaries of convex sets. We prove existence of solutions for a large class of input data and give a uniqueness result. To the author's knowledge, this is the first time that a rigorous mathematical analysis of this problem is given. The approach is based on optimal transportation theory. It yields a practical algorithm for finding the reflectors. Namely, the problem is equivalent to a constrained linear optimization problem.
\end{abstract}

\section{Introduction}

We consider the following beam shaping problem from geometric optics, sketched in Figure 1: Suppose we are given a spherical wavefront with a given intensity distribution emitted from a point source. We would like to transform this input beam into an output beam of parallel light rays with a desired intensity distribution. This transformation is to be achieved with a system of two 
Figure 1: Geometry of the reflector problem. Note our convention regarding spatial coordinates, illustrated by the coordinate system in the lower left hand corner: The output beam propagates in the direction of the negative $z$-axis, and points in the plane perpendicular to the $z$-axis are denoted by the vector $x \in \mathbb{R}^{2}$. Thus a generic point in three dimensional space is denoted by $(x, z)$. For more details see the accompanying text in Section 2.

reflectors ${ }^{1}$. This paper deals with the mathematical problem of finding these reflecting surfaces for given input and output apertures and input and output intensities $^{2}$. The geometric optics approximation is assumed.

These types of problems are of practical interest and there exists an extensive engineering literature on them; see Section 5 in V. Oliker's stimulating survey paper [6] and the many sources cited there. As explained there, the approaches described in the engineering literature are usually only applicable to specific data and are usually only justified by specific numerical examples. In [6], the problem was reformulated as equation of Monge-Ampère type for the polar radius of the first reflector. A rigorous mathematical analysis, showing the existence of solutions, was however lacking as stated in [6].

In the present paper, we provide this rigorous mathematical analysis, using a novel approach to the problem. (To the best of our knowledge, this is the first time such a rigorous analysis has been provided.) We show the existence of solutions for given input and output intensities and provide a uniqueness result for the ray tracing map associated with the reflector system. The two reflectors we obtain are always concave; they are contained in the boundary of certain convex sets. The approach is based on advances in the theory of Monge-Ampère equations and optimal transportation that have been made in about the last 15 years $[1,2,3,10]$. Similar approaches have fruitfully previously been applied to other beam shaping problems by X-J Wang in [12], and independently by V. Oliker and the author in $[5,4]$.

We now describe the approach and results in more detail. For this, denote the input aperture by $\bar{D}$, and the output aperture by $\bar{T}$. Thus $\bar{D}$ is a subset of

\footnotetext{
${ }^{1} \mathrm{~A}$ treatment with three reflectors is possible, see [8]. For reasons of space limitations, a two reflector system is sometimes more desirable from an engineering point of view.

${ }^{2}$ From a practical point of view, an additional requirement is that no blockage of the light may occur. We do not deal with this explicitly, but we note that this can generally be achieved by choosing the reduced optical path length large enough. (See Section 2 for the definition of the reduced optical path length.)
} 
the unit sphere $S^{2}$, and $\bar{T}$ is contained in a plane perpendicular to the direction of the output beam. (See again Figure 1.) The input and output intensities are given by nonnegative functions $I(m), m \in \bar{D}$, and $L(x), x \in \bar{T}$.

We represent the two reflectors via the polar radius $\rho(m), m \in \bar{D}$ for the first one, and as the graph of a function $z(x), x \in \bar{T}$ for the second one. See again Figure 1. One of the main results is that finding the functions $\rho(m)$ and $z(x)$ is equivalent to solving the following constrained optimization problem for certain transforms of $\rho(m)$ and $z(x)$ :

$$
\begin{aligned}
& \text { Minimize } \int_{\bar{D}} \log \hat{\rho}(m) I(m) d \sigma+\int_{\bar{T}} \log \tilde{z}(x) L(x) d x \\
& \text { subject to } \log \hat{\rho}(m)+\log \tilde{z}(x) \geq \log K(m, x) \text { for all } m \in \bar{D}, x \in \bar{T} .
\end{aligned}
$$

Here $\log \hat{\rho}(m)$ and $\log \tilde{z}(x)$ are certain transforms of $\rho(m)$ and $z(x)$, respectively, given explicitly in Definitions 2.1 and 2.2 below. The constraint is given by

$$
\log K(m, x)=\log \left[\frac{\ell-\left\langle m_{x}, x\right\rangle}{2 \ell\left(\ell^{2}-|x|^{2}\right)\left(1+m_{z}\right)}-\frac{1}{4 \ell^{2}}\right] .
$$

Here $m=\left(m_{x}, m_{z}\right) \in \bar{D} \subseteq S^{2}, x \in \bar{T} \subseteq \mathbb{R}^{2}$, and $\ell>0$ is a constant. For details see the text below.

Note that this reformulation not only gives a theoretical existence result for solutions, but it also translates into a practical method for finding the solution. In fact, the discretization of the constrained optimization problem (1)-(2) is a standard linear programming problem and can be solved numerically.

As mentioned before, the approach of this paper has a strong connection to, and is in fact motivated by, the theory of optimal transportation. (See for example $[1,2,3]$, and in general the recent survey [10] and the extremely extensive bibliography cited there.) Consider the ray tracing map, or reflector map, $\gamma: \bar{D} \rightarrow \bar{T}$. So a ray emitted in the direction $m \in \bar{D}$ will be transformed by the reflector system into a ray labeled by $x=\gamma(m) \in \bar{T}$. (See again Figure 1, and also Figure 4.)

Consider the optimal transportation problem for moving the measure $I(m) d \sigma$ on $\bar{D} \subseteq S^{2}$ to the measure $L(x) d x$ on $\bar{T} \subseteq \mathbb{R}^{2}$ via a transformation $P: \bar{D} \rightarrow \bar{T}$ in such a way that the "transportation cost"

$$
C(P)=\int_{\bar{D}} \log K(m, P(m)) I(m) d \sigma
$$

is maximized $^{3}$. We prove that the ray tracing map $\gamma$ actually solves this

\footnotetext{
${ }^{3}$ It is unclear to the author whether the cost $C(P)$ has any physical meaning. If $\max$ -
} 
problem. This gives a uniqueness result for the reflector map, see Theorem 7.3 .

The structure of the paper is as follows: In Section 2, we state the problem and our notation precisely and introduce the transforms $\hat{\rho}(m)$ and $\tilde{z}(m)$. In the next section, we give certain analytical definitions of our concepts of reflectors and the corresponding reflector map. In Section 4, we then show that these analytical constructions correspond to some geometric constructions. This geometric content is that we seek to represent the first reflector as the boundary of the intersection of a certain family of spheroids, and we seek to represent the second reflector as the boundary of the intersection of a family of paraboloids. We also justify that our abstract definition of the reflector map $\gamma$ is consistent with the optical definition. In Section 5, we formulate the reflector problem and show that it is equivalent to the constrained optimization problem (1)-(2). Then in the next section, we prove the existence of solutions, which follows from a standard compactness argument. Finally, in Section 7, we show the connection to the transportation problem mentioned above, and finally state the main theorem, Theorem 7.3, on the existence and uniqueness of solutions to the reflector problem.

Throughout, the treatment is very similar to the papers [5, 4], which treated similar but distinct beam shaping problems. The main difference is that the problem at hand requires the introduction of the transforms $\hat{\rho}(m)$ and $\tilde{z}(x)$, and a more complicated form for the cost function $\log K(m, x)$. These complications arise mostly because the first reflector is best described using polar coordinates, and the second reflector is best described using Cartesian coordinates. For the sake of completeness and being self-contained, we include most of the proofs here, leaving out some details if they can easily be filled in from [5] and [4].

\section{Statement of the problem and assumptions}

We first fix our notations and assumptions in this section. Consider the configuration show in Figure 1. A point source located at the origin $O=(0,0,0)$ generates a spherical wave front over a given input aperture $\bar{D}$ contained in the unit sphere $S^{2}$. The latter is required to be transformed into a beam of parallel rays propagating in the direction of the negative $z$-axis by means of a system of two reflectors. A cross section of the output beam is specified as

imizing a transportation cost seems unintuitive, an equivalent formulation is of course to minimize $-C(P)$ 
a set on a plane perpendicular to the direction of propagation. Such a cross section is called the output aperture, and denoted by $\bar{T}$.

We denote points in space $\mathbb{R}^{3}$ by pairs $(x, z)$, where $x \in \mathbb{R}^{2}$ is the position vector in a plane perpendicular to the direction of propagation and $z \in \mathbb{R}$ is the coordinate in the (negative) direction of propagation. See again Figure 1 for our convention on the direction of the $z$-axis. Points on the unit sphere $S^{2}$ will typically be denoted by $m \in S^{2}$; their components are also written as $m=\left(m_{x}, m_{z}\right)$ with $\left|m_{x}\right|^{2}+m_{z}^{2}=1$.

We fix the output aperture in the plane $z=-d$. We will seek to represent the two reflectors as the graph of the polar radius $\rho(m)$ and as the graph of a function $z(x)$, respectively, as shown in Figure 1. That is

$$
\begin{aligned}
& \text { Reflector 1: } \Gamma_{\rho}=\{\rho(m) \cdot m \mid m \in \bar{D}\}, \\
& \text { Reflector 2: } \Gamma_{z}=\{(x, z(x)) \mid x \in \bar{T}\} .
\end{aligned}
$$

We now have the following assumptions on the input data. Let $D$ be an open, nonempty subset of $S^{2}$ with closure $\bar{D}$ such that $(0,0,1) \notin \bar{D}$ and $(0,0,-1) \notin \bar{D}$. So there is some $\varepsilon>0$ such that for $m=\left(m_{x}, m_{z}\right) \in \bar{D}$, we have $1-\varepsilon>m_{z}>-1+\varepsilon$. Let further $T$ to be an open, bounded, nonempty subset of $\mathbb{R}^{2}$. Denote its closure by $\bar{T}$.

The geometrical optics approximation is assumed. It follows from general principles of geometric optics that all rays will have equal length from $(0,0,0)$ to the plane $z=-d$; this length is called the optical path length and will be denoted by $L$. We define the reduced optical path length as $\ell=L-d$. In terms of the mathematical problem, $\ell$ is an input parameter. We pick the reduced optical path length $\ell>0$ large enough so that the following conditions are satisfied:

$$
\begin{aligned}
& \ell>\max _{x \in \bar{T}}|x|, \quad \text { and } \\
& \frac{\ell-\left\langle m_{x}, x\right\rangle}{\left(\ell^{2}-|x|^{2}\right)\left(1+m_{z}\right)}-\frac{1}{2 \ell}>0 \text { for all } m \in \bar{D}, x \in \bar{T} .
\end{aligned}
$$

(It is not hard to see that the second condition is indeed satisfied for large enough $\ell$.)

We'll also use the convenient notation

$$
\delta=\frac{1}{2 \ell} .
$$

Finally, we define the following two transformations, which are central to the analysis: 
Definition 2.1. Let $z=z(x)$ be a continuous function defined on $\bar{T} \subseteq \mathbb{R}^{2}$. Then define the function

$$
\tilde{z}(x)=\delta-\frac{z(x)}{\ell^{2}-|x|^{2}} \quad \text { for } x \in \bar{T} .
$$

Definition 2.2. Let $\rho=\rho(m)$ be a continuous function defined on $\bar{D} \subseteq S^{2}$ with $\rho>0$. Then define the function

$$
\hat{\rho}(m)=-\delta+\frac{1}{2 \rho(m) \cdot\left(m_{z}+1\right)} \quad \text { for } m \in \bar{D} .
$$

These transformations are obviously invertible, namely

$$
\begin{array}{lr}
z(x)=(-\tilde{z}(x)+\delta)\left(\ell^{2}-|x|^{2}\right) & \text { for } x \in \bar{T}, \\
\rho(m)=\left(2\left(m_{z}+1\right)(\hat{\rho}(m)+\delta)\right)^{-1} & \text { for } m \in \bar{D} .
\end{array}
$$

The following lemma is now obvious with the above formulas.

Lemma 2.3. (i) The transformation $C(\bar{T}) \rightarrow C(\bar{T}), z \mapsto \tilde{z}$, is a bijection.

(ii) Denote by $C_{>0}(\bar{D})$ the set of all positive continuous functions on $\bar{D}$. Then the transformation $C_{>0}(\bar{D}) \rightarrow\{\sigma \in C(\bar{D}) \mid \sigma>-\delta\}, \rho \mapsto \hat{\rho}$, is a bijection.

\section{Reflector pairs and the reflector map}

We now come to the central definition of this paper, namely that of a reflector pair. We first give the analytic definition. In the next section, we look at the geometric interpretation. In preparation, we first define the following function:

Definition 3.1. Define the function

$$
K(m, x)=\delta \frac{\ell-\left\langle m_{x}, x\right\rangle}{\left(\ell^{2}-|x|^{2}\right)\left(1+m_{z}\right)}-\delta^{2} \text { for } m=\left(m_{x}, m_{z}\right) \in \bar{D}, x \in \bar{T} .
$$

Note that $K(m, x)>0$ by (4). The function $\log K(m, x)$ can be interpreted as some kind of cost of transporting a unit of energy from $m$ to $x$. (See Section 7 for more details.)

We are now ready to define the notion of a reflector pair. 
Definition 3.2. A pair $(\rho, z) \in C_{>0}(\bar{D}) \times C(\bar{T})$ is called a reflector pair if $\hat{\rho}, \tilde{z}>0$ and

$$
\begin{aligned}
\hat{\rho}(m) & =\sup _{x \in \bar{T}}\left(\frac{1}{\tilde{z}(x)} K(m, x)\right) \text { for } m \in \bar{D}, \\
\tilde{z}(x) & =\sup _{m \in \bar{D}}\left(\frac{1}{\hat{\rho}(m)} K(m, x)\right) \text { for } x \in \bar{T} .
\end{aligned}
$$

Here we used the definitions of $\hat{\rho}$ and $\tilde{z}$ from Definitions 2.2 and 2.1. Note that the suprema on the right hand sides are in fact attained. Also note that for a reflector pair $(\rho, z)$, we have

$$
\hat{\rho}(m) \tilde{z}(x) \geq K(m, x) \quad \text { for all } m \in \bar{D}, x \in \bar{T} .
$$

We will show later that reflector pairs are in fact concave (see Proposition 4.8) and that $\log \hat{\rho}$ and $\log \tilde{z}$ are uniformly Lipschitz continuous (see Proposition 6.2).

Finally, we define the reflector map, or ray-tracing map, associated to a reflector pair. Again, the choice of terminology will become clear when we consider the problem from a geometric viewpoint in Section 4.4.

Definition 3.3. Let $(\rho, z) \in C_{>0}(\bar{D}) \times C(\bar{T})$ be a reflector pair. Define its reflector map, or ray tracing map, as a set-valued map $\gamma: \bar{D} \rightarrow\{$ subsets of $\bar{T}\}$ via

$$
\gamma(m)=\left\{x \in \bar{T} \mid \hat{\rho}(m)=\frac{1}{\tilde{z}(x)} K(m, x)\right\} \quad \text { for } m \in \bar{D} .
$$

Clearly $\gamma(m) \neq \emptyset$ for any $m \in \bar{D}$ by $(7)$. We will show later that $\gamma(m)$ is in fact single-valued for almost all $m \in \bar{D}$. (See Proposition 4.10.) We may thus regard $\gamma$ as a transformation $\gamma: \bar{D} \rightarrow \bar{T}$.

\section{Reflector pairs: Geometric viewpoint}

In this section, we will investigate the definition of reflector pairs from a geometric point of view. We show that the reflectors can be obtained as the boundary of certain convex sets. These sets in turn are the intersections of a family of spheroids (for reflector 1) and paraboloids (for reflector 2), respectively.

In the first two sections, we will use the following notation, the pointwise analogue of Definitions 2.1 and 2.2: For $x \in \mathbb{R}^{2}$ and $z>0$, and $m \in S^{2}$ and 
Figure 2: LEFT: The set $S_{x, z}$ is a spheroid with foci at the origin $O$ and at $(x, z) . \bar{S}_{x, z}$ is the convex set bounded by $S_{x, z}$. The sketch shows a cross section through a plane containing the axis defined by the foci. The sets $S_{x, z}$ and $\bar{S}_{x, z}$ are obtained by rotating the gray ellipse around the axis through the foci as indicated. RIGHT: The set $P_{x, z}$ is a paraboloid with focus at $\rho \cdot m$ and axis parallel to the $z$-axis. $\bar{P}_{\rho \cdot m}$ is the convex set bounded by $P_{\rho \cdot m}$. The sketch shows a cross section through a plane containing the axis and the focus. The sets $P_{\rho \cdot m}$ and $\bar{P}_{\rho \cdot m}$ are obtained by rotating the gray parabola around the axis as indicated.

$\rho>0$, write

$$
\begin{aligned}
& \tilde{z}=\delta-\frac{z}{\ell^{2}-|x|^{2}} \\
& \hat{\rho}=-\delta+\frac{1}{2 \rho\left(m_{z}+1\right)} .
\end{aligned}
$$

\subsection{The spheroids $S_{x, z}$}

We define first a family of spheroids, indexed by points $(x, z)$.

Definition 4.1. Let $x \in \bar{T}$ and $z \in \mathbb{R}$ such that $\tilde{z}>0$. Define the set

$$
S_{x, z}=\left\{\rho \cdot m \mid m \in S^{2}, \hat{\rho}=\frac{1}{\tilde{z}} K(m, x)\right\} .
$$

Here we used the notation from (10) and (11).

Lemma 4.2. Using the notation from the previous definition, the condition $\tilde{z}>0$ implies $\ell-z>|(x, z)|$. (Here $|$.$| denotes the standard Euclidean vector$ norm.) The set $S_{x, z}$ is given by the following equation for $\rho>0, m \in S^{2}$ :

$$
\rho+|(x, z)-\rho \cdot m|=\ell-z .
$$

Geometrically, $S_{x, z}$ is a spheroid whose foci are the origin $O=(0,0,0)$ and the point $(x, z)$. (See Figure 2.)

Proof. Note first that the condition $\tilde{z}>0$ implies

$$
z=(\delta-\tilde{z})\left(\ell^{2}-|x|^{2}\right)<\delta\left(\ell^{2}-|x|^{2}\right)=\frac{\ell}{2}-\frac{|x|^{2}}{2 \ell},
$$


and so

$$
\ell-z>\frac{\ell}{2}+\frac{|x|^{2}}{2 \ell}>0
$$

Also,

$$
(\ell-z)^{2}=\ell^{2}+z^{2}-2 \ell z>z^{2}+|x|^{2}=|(x, z)|^{2} .
$$

It follows that

$$
\ell-z>|(x, z)| \text {. }
$$

A straightforward but lengthy algebraic computation now yields that the condition in (12) is equivalent to

$$
(\ell-z-\rho)^{2}=|(x, z)-\rho \cdot m|^{2} .
$$

Here $\rho=\left(2\left(m_{z}+1\right)(\hat{\rho}+\delta)\right)^{-1}>0$, as $\hat{\rho}>0$. We have

$$
\ell-z>|(x, z)| \geq \rho-|(x, z)-\rho \cdot m|=\rho-|\ell-z-\rho|,
$$

and thus $\ell-z-\rho>-|\ell-z-\rho|$. It follows that

$$
\ell-z-\rho=|\ell-z-\rho| .
$$

This together with (14) gives equation (13). Now consider the geometric content of equation (13). We can immediately read off that the set $S_{x, z}$ consists of all points that satisfy that the sum of the distances to the points $(0,0,0)$ and $(x, z)$ equals $\ell-z$. (See Figure 2. Also note that $\ell-z>|(x, z)|$ implies that the set $S_{x, z}$ is nonempty.) This is by definition a spheroid.

Definition 4.3. Let as before $x \in \mathbb{R}^{2}$ and $z \in \mathbb{R}$ such that $\tilde{z}>0$. Denote by $\bar{S}_{x, z}$ the closed convex set bounded by $S_{x, z}$. Thus $\bar{S}_{x, z}$ is given by

$$
\bar{S}_{(x, z)}=\left\{\rho \cdot m \mid m \in S^{2}, \hat{\rho} \geq \frac{1}{\tilde{z}} K(m, x)\right\} .
$$

\subsection{The paraboloids $P_{\rho \cdot m}$}

Similarly to the previous section, we now define a family of paraboloids $P_{\rho \cdot m}$.

Definition 4.4. Let $m \in \bar{D} \subseteq S^{2}$ and $\rho>0$ such that $\hat{\rho}>0$. Define the set

$$
P_{\rho \cdot m}=\left\{(x, z) \mid x \in \mathbb{R}^{2}, \tilde{z}=\frac{1}{\hat{\rho}} K(m, x)\right\} .
$$

Here we used again the notation (10), (11). 
Lemma 4.5. With the notation of the previous definition, consider new coordinates $(p, q)$ obtained by a shift by $\rho \cdot m$, that is $(p, q)=(x, z)-\rho \cdot m$. Then the set $P_{\rho \cdot m}$ is given by the equation

$$
4 a q=|p|^{2}-4 a^{2}
$$

where

$$
a=\frac{2 \rho \delta(1+m z)-1}{4 \delta}<0 .
$$

Geometrically, $P_{\rho \cdot m}$ is a paraboloid with focus at $\rho \cdot m$ and axis parallel to the $z$-axis. It opens in the directions of the negative $z$-axis. (See Figure 2.)

Proof. A straightforward computation yields that (16) is equivalent to (17). Note that a paraboloid with focus at $(0,0,0)$ and focal parameter $2 \alpha$ has the equation $4 \alpha z=|x|^{2}-4 \alpha^{2}$. Also note that $a<0$ follows from $\hat{\rho}>0$.

Definition 4.6. Let as before $m \in \bar{D} \subseteq S^{2}$ and $\rho>0$ such that $\hat{\rho}>0$. Denote by $\bar{P}_{\rho \cdot m}$ the closed convex set bounded by $P_{\rho \cdot m}$. Thus $\bar{P}_{\rho \cdot m}$ is given by

$$
\bar{P}_{\rho \cdot m}=\left\{(x, z) \mid x \in \mathbb{R}^{2}, \tilde{z} \geq \frac{1}{\hat{\rho}} K(m, x)\right\} .
$$

\subsection{Geometry of reflector pairs}

We now investigate the geometric content of the definition of reflector pairs. For this, suppose that $(\rho, z) \in C_{>0}(\bar{D}) \times C(\bar{T})$ is a reflector pair. Consider the two sets

$$
\begin{aligned}
& \bar{S}=\bigcap_{x \in \bar{T}} \bar{S}_{x, z(x)}, \\
& \bar{P}=\bigcap_{m \in \bar{D}} \bar{P}_{\rho(m) m} .
\end{aligned}
$$

Note that none of the sets $\bar{S}_{x, z(x)}$ and $\bar{P}_{\rho(m) m}$ are empty as in fact $\hat{\rho}(m)>0$ for all $m \in \bar{D}$ by definition of reflector pairs and $\tilde{z}(x)>0$ for all $x \in \bar{T}$. A compactness argument yields that $\bar{P}$ and $\bar{S}$ are nonempty as well, and they are convex sets, since they are the intersections of convex sets. Moreover, the algebraic representations (15) and (18) along with the definition of reflector pairs immediately give rise to the following geometric facts:

Proposition 4.7. Let $(\rho, z) \in C_{>0}(\bar{D}) \times C(\bar{T})$ be a reflector pair. 
Figure 3: Geometry of reflector pairs. LEFT: The graph $\Gamma_{\rho}$ of $\rho(m)$ is obtained by intersecting the boundary of the convex set $\bar{S}$ with the cone $\mathbb{R}^{+} \bar{D}$. RIGHT: The graph $\Gamma_{z}$ of $z(x)$ is obtained by intersecting the boundary of the convex set $\bar{P}$ with the cylinder $\bar{T} \times \mathbb{R}$.

(i) The graph of $\rho$,

$$
\Gamma_{\rho}=\{\rho(m) \cdot m \mid m \in \bar{D}\},
$$

is contained in the boundary $\partial \bar{S}$ of the convex set $\bar{S}$ given in (19). In fact,

$$
\Gamma_{\rho}=\partial \bar{S} \cap\left(\mathbb{R}^{+} \cdot \bar{D}\right),
$$

where $\mathbb{R}^{+} \cdot \bar{D}=\{r \cdot m \mid r \geq 0, m \in \bar{D}\}$ is a cone with vertex at the origin and cross section $\bar{D}$. (See Figure 3.)

(ii) The graph of $\rho$,

$$
\Gamma_{z}=\{(x, z(x)) \mid x \in \bar{T}\}
$$

is contained in the boundary $\partial \bar{P}$ of the convex set $\bar{P}$ given in (20). In fact,

$$
\Gamma_{z}=\partial \bar{P} \cap(\bar{T} \times \mathbb{R}),
$$

where $\bar{T} \times \mathbb{R}=\{(x, h) \mid x \in \bar{T}, h \in \mathbb{R}\}$ is the cylinder with base $\bar{T}$. (See Figure 3.)

We may thus think of the two reflectors as the "envelopes" of certain families of spheroids and paraboloids, respectively. An immediate corollary is also the following result:

Proposition 4.8. If $(\rho, z) \in C_{>0}(\bar{D}) \times C(\bar{T})$ is a reflector pair, then $z$ is a concave function and $\rho$ is the radial function of a convex set. In particular, both $\rho$ and $z$ are locally Lipschitz continuous and almost everywhere differentiable. (Here "almost everywhere" refers to the standard Lebesgue measures on $S^{2}$ and $\mathbb{R}^{2}$, respectively.)

Proof. The first part of the above statement follows immediately from the previous discussion. The Lipschitz and differentiablility properties are standard results from convexity theories, see [9], Theorem 1.5.1 and Section 1.7. 


\subsection{Geometry of the reflector map}

We now investigate the geometry of the reflector map. For this, the following terminology is useful: Suppose $(\rho, z) \in C_{>0}(\bar{D}) \times C(\bar{T})$ is a reflector pair. For $m \in \bar{D}$, if $\rho(m) \cdot m \in \Gamma_{\rho} \cap S_{x, z(x)}$, say that the spheroid $S_{x, z(x)}$ is supporting to the graph $\Gamma_{\rho}$ at the point $\rho(m) \cdot m$.

Similarly, for $x \in \bar{T}$, if $(x, z(x)) \in \Gamma_{z} \cap P_{\rho(m) \cdot m}$, say the paraboloid $P_{\rho(m) \cdot m}$ is supporting to $\Gamma_{z}$ at $(x, z(x))$.

Note that $S_{x, z(x)}$ is supporting to $\Gamma_{\rho}$ at the point $\rho(m) \cdot m$ if and only if $P_{\rho(m) \cdot m}$ is supporting to $\Gamma_{z}$ at $(x, z(x))$. This is because both statements are equivalent to

$$
\tilde{z}(x) \hat{\rho}(m)=K(m, x)
$$

by the definitions (16) and (12).

We also have the following geometric lemma:

Lemma 4.9. Let $x_{1}, x_{2} \in \bar{T}$ be two distinct point: $x_{1} \neq x_{2}$. Suppose the spheroids $S_{x_{1}, z\left(x_{1}\right)}$ and $S_{x_{2}, z\left(x_{2}\right)}$ are supporting to $\Gamma_{\rho}$ at the same point $\rho(m) \cdot m$. Then $S_{x_{1}, z\left(x_{1}\right)}$ and $S_{x_{2}, z\left(x_{2}\right)}$ intersect tranversally at $\rho(m) \cdot m$.

Proof. Assume the contrary, that is, that $S_{x_{1}, z\left(x_{1}\right)}$ and $S_{x_{2}, z\left(x_{2}\right)}$ intersect tangentially to each other. Since the two spheroids share the focus $O$, it follows from basic properties of ellipsoids that the two line segments $\overline{\left(x_{1}, z\left(x_{1}\right)\right), \rho(m) \cdot m}$ and $\overline{\left(x_{2}, z\left(x_{2}\right)\right), \rho(m) \cdot m}$ are parallel. Thus the three points $\left(x_{1}, z\left(x_{1}\right)\right),\left(x_{2}, z\left(x_{2}\right)\right)$ and $\rho(m) \cdot m$ are collinear. On the axis through these three points, the point $\rho(m) \cdot m$ cannot lie between the other two points; that is $\rho(m) \cdot m$ must be one of the end points of the line segment defined by the three points. But this contradicts that the paraboloid $P_{\rho(m) \cdot m}$ contains both $\left(x_{1}, z\left(x_{1}\right)\right)$ and $\left(x_{2}, z\left(x_{2}\right)\right)$.

Now consider the reflector map $\gamma$ associated with a reflector pair $(\rho, z)$ as defined in Definition 3.3. In the language defined above, we may now say that $\gamma(m)$ is the set of all points $x \in \bar{T}$ such that that the spheroid $S_{x, z(x)}$ is supporting to the graph $\Gamma_{\rho}$ at the point $\rho(m) \cdot m$. Consider the case where for some $m \in \bar{D}$, the set $\gamma(m)$ contains more than one point, say $\left\{x_{1}, x_{2}\right\} \subseteq \gamma(m)$. By the previous lemma, the two spheroids $S_{x_{1}, z\left(x_{1}\right)}$ and $S_{x_{2}, z\left(x_{2}\right)}$ intersect transversally at $\rho(m) \cdot m$. Thus $\rho$ is not differentiable at $m$. But the set of such points has measure zero by Proposition 4.8. We thus immediately have the following result:

Proposition 4.10. Let $(\rho, z) \in C_{>0}(\bar{D}) \times C(\bar{T})$ be a reflector pair. Then its reflector map $\gamma: \bar{D} \rightarrow \bar{T}$ is almost everywhere single-valued. That is, the set of points $m$ where $\gamma(m)$ is not single valued has measure zero with respect to the 
Figure 4: Geometry of the reflector map: A ray emitted from the origin $O$ will be reflected to a ray traveling in the negative $z$-direction labeled by $x=\gamma(x)$. See the text for details.

standard measure on $S^{2}$ as a submanifold of the measure space $\left(\mathbb{R}^{3}, \mu\right)$, where $\mu$ is the standard Lebesgue measure.

Remark 4.11. We now justify the terminology of the reflector map from an optical point of view. See Figure 4 for the following considerations. Let $(\rho, z) \in C_{>0}(\bar{D}) \times C(\bar{T})$ be a reflector pair. Let $m \in \bar{D}$ such that $\gamma(m)$ is single valued. We show that under the geometric optics approximation, a ray emitted in the direction $m \in \bar{D}$ will be reflected off the first reflector $\Gamma_{\rho}$ and then the second reflector $\Gamma_{z}$ in such a way that the reflected ray is parallel to the negative $z$-axis and that it intersects a plane perpendicular to the $z$-axis in the point $x=\gamma(m)$.

Consider the reflection off reflector 1 first. Since the spheroid $S_{x, z(x)}$ is tangential to the reflector $\Gamma_{\rho}$, the ray will be reflected off $\Gamma_{\rho}$ the same way it would be reflected off $S_{x, z(x)}$. By the geometrical properties of spheroids, this means that the ray is reflected towards the focus $(x, z(x))$. There, the ray will encounter $\Gamma_{z}$. It will be reflected the same way as it would be reflected off the paraboloid $P_{\rho(m) \cdot m}$, that is, in the direction of the negative $z$-axis.

Thus our definition of the reflector map is in agreement with the physical law of reflection. In the case when $\gamma(m)$ is multi-valued, the first reflector has a singular point and a ray will split up into a cone of light rays. These rays will generate a set of directions whose projection onto a plane perpendicular to the $z$-axis is $\gamma(m)$. This is consistent with the physical phenomenon of diffraction at singularities.

The following statements about the reflector map $\gamma(m)$ are analogous to Theorem 4.8 and Lemma 4.9 in [5]. See this paper and the the reference [7] for further details on the proofs.

Theorem 4.12. Let $\mathcal{B}$ denote the $\sigma$ algebra of Borel sets on $\bar{T}$. Then for any subset $\tau \in \mathcal{B}, \gamma^{-1}(\tau)$ is measurable relative to the standard Lebesgue measure of $\bar{D}$. In addition, for any non-negative locally integrable function $I$ on $\bar{D}$, the function

$$
\mathcal{L}(\tau)=\int_{\gamma^{-1}(\tau)} I(m) d \sigma
$$


is a non-negative completely additive measure on $\mathcal{B}$. (Here do is the standard measure on $S^{2} \subseteq \mathbb{R}^{3}$.)

Lemma 4.13. With the notation of the above Theorem, let $h$ be a continuous function on $\bar{T}$. Then we have

$$
\int_{\bar{T}} h(x) \mathcal{L}(d x)=\int_{\bar{D}} h(\gamma(m)) I(m) d \sigma .
$$

\section{The reflector problem and an equivalent con- strained minimization problem}

Let now $I$ be a nonnegative, integrable function on $\bar{D}$, and $L$ be a nonnegative, integrable function on $\bar{T}$, such that

$$
\int_{\bar{D}} I(m) d \sigma=\int_{\bar{T}} L(x) d x .
$$

We may interpret $I$ and $L$ as the intensity distribution functions of the light beams on the input and output apertures, respectively. The above integral condition is simply (total) energy conservation.

In this section of the paper, we now formulate the reflector problem. More specifically, we can call this formulation a "weak" version of the reflector problem since we do not require the input functions $I$ and $L$ to be differentiable, nor do we require the reflectors to be smooth surfaces.

We then formulate a second problem, which is an infinite dimensional linear programming problem. One of the main results is that the two problems are in fact equivalent. This is stated and proved at the end of this section.

Let us first formulate the reflector problem:

Problem I. (Reflector Problem) For given input and output intensities I and $L$ satisfying (21), find a pair $(z, \rho) \in C_{>0}(\bar{D}) \times C(\bar{T})$ that satisfies the following conditions:

(i) $(z, \rho)$ is a reflector pair

(ii) The reflector map $\gamma: \bar{D} \rightarrow \bar{T}$ satisfies

$$
\int_{\gamma^{-1}(\tau)} I(m) d \sigma=\int_{\tau} L(x) d x
$$

for any Borel set $\tau \subseteq \bar{T}$. 
This formulation builds on the geometrical interpretation of reflector maps as presented in Section 4. Note that condition (ii) is local energy conservation.

We have the following immediate corollary from Lemma 4.13:

Corollary 5.1. Let $(z, \rho)$ be a solution to Problem I. Then we have

$$
\int_{\bar{D}} h(\gamma(m)) I(m) d \sigma=\int_{\bar{T}} h(x) L(x) d x
$$

for all functions $h \in C(\bar{T})$.

Before we now formulate Problem II, we define the following function space:

Definition 5.2. Define the set of admissible functions as

$$
\begin{aligned}
\operatorname{Adm}(\bar{D}, \bar{T})=\{(r, \zeta) \in C(\bar{D}) \times C(\bar{T}) \mid r(m)+\zeta(x) & \geq \log K(m, x) \\
& \text { for all } m \in \bar{D}, x \in \bar{T}\} .
\end{aligned}
$$

Problem II. Minimize the functional

$$
\mathcal{F}(r, \zeta)=\int_{\bar{D}} r(m) I(m) d \sigma+\int_{\bar{T}} \zeta(x) L(x) d x
$$

on the space $\operatorname{Adm}(\bar{D}, \bar{T})$.

The two problems are equivalent, as expressed in the following theorem.

Theorem 5.3. Let $(\rho, z) \in C_{>0}(\bar{D}) \times C(\bar{T})$ be a reflector pair. Then $(\log \hat{\rho}, \log \tilde{z}) \in$ $\operatorname{Adm}(\bar{D}, \bar{T})$. The following statements are equivalent:

(i) $(\rho, z)$ solves the Reflector Problem I.

(ii) $(\log \hat{\rho}, \log \tilde{z})$ minimizes the functional $\mathcal{F}$ on $\operatorname{Adm}(\bar{D}, \bar{T})$.

Proof. The statement $(\log \hat{\rho}, \log \tilde{z}) \in \operatorname{Adm}(\bar{D}, \bar{T})$ follows immediately from (9). The proof of the equivalence of (i) and (ii) is analogous to those of Theorem 5.2 in [5] and Theorem 3.4 in [4]. See also Theorem 1 in [3]. Since this theorem is central to this paper, we give an outline of the proof, omitting some of the technicalities, which can be filled in with the above references.

$(i) \Rightarrow(i i)$ : Suppose $(\rho, z)$ solves Problem I with corresponding reflector map $\gamma$. Let $(r, \zeta) \in \operatorname{Adm}(\bar{D}, \bar{T})$. Then for any $m \in \bar{D}$ such that $\gamma(m)$ is single-valued, we have

$$
r(m)+\zeta(\gamma(m)) \geq \log K(m, \gamma(m))=\log \hat{\rho}(m)+\log \tilde{z}(\gamma(m)) .
$$


This yields

$$
\begin{aligned}
\int_{\bar{D}} r(m) I(m) d \sigma+ & \int_{\bar{D}} \zeta(\gamma(m)) I(m) d \sigma \\
& \geq \int_{\bar{D}} \log \hat{\rho}(m) I(m) d \sigma+\int_{\bar{D}} \log \tilde{z}(\gamma(m)) I(m) d \sigma .
\end{aligned}
$$

Now using Corollary 5.1 gives $\mathcal{F}(r, \zeta) \geq \mathcal{F}(\log \tilde{z}, \log \hat{\rho})$.

$($ ii $) \Rightarrow(i)$ : The main idea is that the Euler-Lagrange equations of minimizing $\mathcal{F}$ are equivalent to the equality

$$
\int_{\bar{D}} h(\gamma(m)) I(m) d \sigma=\int_{\bar{T}} h(x) L(x) d x
$$

for all functions $h \in C(\bar{T})$. This implies that $(\rho, z)$ solves Problem I.

Let thus $h \in C(\bar{T})$. Let $\varepsilon>0$ be a small parameter. To bring out the main ideas, we present a formal calculation, assuming expansions in $\varepsilon$ are valid. A completely rigorous treatment is possible; indeed the proof in [5] can easily be modified to the problem at hand.

Define perturbations $\left(r_{\varepsilon}, \zeta_{\varepsilon}\right) \in \operatorname{Adm}(\bar{D}, \bar{T})$ of $(\log \hat{\rho}, \log \tilde{z})$ via

$$
\begin{aligned}
\zeta_{\varepsilon}(x) & =\log \tilde{z}(x)+\varepsilon h(x) \quad \text { for } x \in \bar{T} \\
r_{\varepsilon}(m) & =\sup _{x \in \bar{T}}\left(-\zeta_{\varepsilon}(x)+\log K(m, x)\right) \quad \text { for } m \in \bar{D} .
\end{aligned}
$$

Let now $x_{\varepsilon}$ be a point where the supremum in the definition of $r_{\varepsilon}(m)$ is attained. Expanding $x_{\varepsilon}$ in $\varepsilon$ yields

$$
x_{\varepsilon}=\gamma(m)+\mathcal{O}(\varepsilon) .
$$

Thus again an expansion in $\varepsilon$ gives

$$
\begin{aligned}
r_{\varepsilon}(m) & =-\zeta_{\varepsilon}\left(x_{\varepsilon}\right)+\log K\left(m, x_{\varepsilon}\right) \\
& =-\log \tilde{z}(\gamma(m))+\log K(m, \gamma(m))-\varepsilon h(\gamma(m))+\mathcal{O}\left(\varepsilon^{2}\right) \\
& =\log \hat{\rho}(m)-\varepsilon h(\gamma(m))+\mathcal{O}\left(\varepsilon^{2}\right) .
\end{aligned}
$$

Thus, using the fact that $(\log \hat{\rho}, \log \tilde{z})$ minimizes $\mathcal{F}$,

$$
0=\left.\frac{d}{d \varepsilon}\right|_{\varepsilon=0} \mathcal{F}\left(r_{\varepsilon}, \zeta_{\varepsilon}\right)=-\int_{\bar{D}} h(\gamma(m)) I(m) d \sigma+\int_{\bar{T}} h(x) L(x) d x .
$$

This completes the sketch of the proof. 


\section{$6 \quad$ Existence of solutions}

Note that Theorem 5.3, while showing that Problems I and II are equivalent, does not state that solutions exist. We prove this in the present section.

In the following, fix some point $m^{*} \in \bar{D}$.

Definition 6.1. Set

$$
\begin{aligned}
\operatorname{Adm}_{\mathrm{refl}}(\bar{D}, \bar{T})=\{(\log \hat{\rho}, \log \tilde{z}) & \in C(\bar{D}) \times C(\bar{T}) \mid \\
& \left.(\rho, z) \text { is a reflector pair with } \log \hat{\rho}\left(m^{*}\right)=0\right\} .
\end{aligned}
$$

Note that $\operatorname{Adm}_{\text {refl }}(\bar{D}, \bar{T}) \subseteq \operatorname{Adm}(\bar{D}, \bar{T})$.

Proposition 6.2. The family of pairs of functions $\operatorname{Adm}_{\text {refl }}(\bar{D}, \bar{T})$ is uniformly Lipschitz continuous in each entry. That is, there are constants $K_{1}, K_{2}>0$ such that

$$
\begin{aligned}
& \left|\zeta\left(x_{1}\right)-\zeta\left(x_{2}\right)\right| \leq K_{1} \cdot\left|x_{1}-x_{2}\right| \\
& \left|r\left(m_{1}\right)-r\left(m_{2}\right)\right| \leq K_{2} \cdot d\left(m_{1}, m_{2}\right)
\end{aligned}
$$

for all $(r, \zeta) \in \operatorname{Adm}_{\text {refl }}(\bar{D}, \bar{T}), x_{1}, x_{2} \in \bar{T}, m_{1}, m_{2} \in \bar{D}$. Here $d\left(m_{1}, m_{2}\right)=$ dist $_{S^{2}}\left(m_{1}, m_{2}\right)$ is the intrinsic distance on $S^{2}$. The constants $K_{1}, K_{2}$ only depend on $\bar{D}, \bar{T}$; explicitly, we have

$$
\begin{aligned}
& K_{1}=\max _{m \in \bar{D}, x \in \bar{T}}\left|\nabla_{x} \log K(m, x)\right|, \\
& K_{2}=\max _{m \in \bar{D}, x \in \bar{T}}\left|\nabla_{m} \log K(m, x)\right| .
\end{aligned}
$$

(Here $\nabla_{m}$ denotes the gradient with respect to the variable $m$ on the sphere $S^{2}$, and $\nabla_{x}$ is the gradient with respect to $x$.)

Proof. We prove the inequality for $\zeta$; the proof of the other inequality is completely analogous. Let $x_{1}, x_{2} \in \bar{T}$ and $(r, \zeta) \in \operatorname{Adm}_{\text {refl }}(\bar{D}, \bar{T})$. Assume $\zeta\left(x_{2}\right) \leq$ $\zeta\left(x_{1}\right)$, otherwise relabel $x_{1}, x_{2}$. Let $m_{1} \in \bar{D}$ be such that $\zeta\left(x_{1}\right)+r\left(m_{1}\right)=$ $\log K\left(m_{1}, x_{1}\right)$. (Such an $m_{1}$ exists by (8).) Then

$$
\begin{aligned}
0 \leq \zeta\left(x_{1}\right)-\zeta\left(x_{2}\right) & =-\zeta\left(x_{2}\right)-r\left(m_{1}\right)+\log K\left(m_{1}, x_{1}\right) \\
& \leq \log K\left(m_{1}, x_{1}\right)-\log K\left(m_{1}, x_{2}\right) \\
& \leq\left(\max _{m \in \bar{D}, x \in \bar{T}}\left|\nabla_{x} \log K(m, x)\right|\right) \cdot\left|x_{1}-x_{2}\right|
\end{aligned}
$$


Proposition 6.3. Functions in $\operatorname{Adm}_{\text {refl }}(\bar{D}, \bar{T})$ are uniformly bounded. Specifically, we have

$$
\begin{aligned}
& |r(m)| \leq K_{1} \operatorname{diam}(\bar{D}) \\
& |\zeta(x)| \leq \max _{x^{\prime} \in \bar{T}}\left|\log K\left(m^{*}, x^{\prime}\right)\right|+K_{2} \operatorname{diam}(\bar{T}) .
\end{aligned}
$$

for all $(r, \zeta) \in \operatorname{Adm}_{\text {refl }}(\bar{D}, \bar{T}), x \in \bar{T}, m \in \bar{D}$. Here $K_{1}, K_{2}$ are as in Proposition 6.2, and $\operatorname{diam}(\bar{T})=\max _{x_{1}, x_{2} \in \bar{T}}\left|x_{1}-x_{2}\right|$ and $\operatorname{diam}(\bar{D})=\max _{m_{1}, m_{2} \in \bar{D}} d\left(m_{1}, m_{2}\right)$ are the diameters of $\bar{T}$ and $\bar{D}$, respectively.

Proof. Let $(r, \zeta) \in \operatorname{Adm}_{\text {refl }}(\bar{D}, \bar{T}), x \in \bar{T}, m \in \bar{D}$. By Proposition 6.2, we have

$$
|r(m)| \leq\left|r\left(m^{*}\right)\right|+K_{1} d\left(m, m^{*}\right) \leq K_{1} \operatorname{diam}(\bar{D}) .
$$

Also, for some $x^{*} \in \gamma\left(m^{*}\right)$, we have $\zeta\left(x^{*}\right)=\log K\left(m^{*}, x^{*}\right)$, and thus again by Proposition 6.2,

$$
|\zeta(x)| \leq\left|\zeta\left(x^{*}\right)\right|+K_{2}\left|x-x^{*}\right| \leq \max _{x^{\prime} \in \bar{T}}\left|\log K\left(m^{*}, x^{\prime}\right)\right|+K_{2} \operatorname{diam}(\bar{T}) .
$$

We can now prove the existence of a solution to Problem II. With Theorem 5.3, this immediately implies the existence of a solution to the reflector problem.

Theorem 6.4. The functional $\mathcal{F}$ attains its minimum on $\operatorname{Adm}(\bar{D}, \bar{T})$. Moreover, this minimum is actually attained at some $(r, \zeta) \in \operatorname{Adm}_{\text {refl }}(\bar{D}, \bar{T})$.

Corollary 6.5. The Reflector Problem I has a solution.

Proof. By Propositions 6.2 and 6.3 and the Arzelà-Ascoli Theorem, the functional $\mathcal{F}$ attains its minimum on $\operatorname{Adm}_{\text {reff }}(\bar{D}, \bar{T})$.

We now show that this minimum is also the minimum of $\mathcal{F}$ on the larger set $\operatorname{Adm}(\bar{D}, \bar{T})$. For this, let $(r, \zeta) \in \operatorname{Adm}(\bar{D}, \bar{T})$. Define

$$
\begin{array}{ll}
r^{*}(m)=\sup _{x \in \bar{T}}\{-\zeta(x)+\log K(m, x)\} & \text { for } m \in \bar{D} \\
\zeta^{*}(x)=\sup _{m \in \bar{D}}\left\{-r^{*}(m)+\log K(m, x)\right\} & \text { for } x \in \bar{T}
\end{array}
$$

It follows that

$$
r^{*}(m) \leq r(m), \quad \zeta^{*}(x) \leq \zeta(x)
$$


for all $m \in \bar{D}, x \in \bar{T}$. Note that $\zeta^{*}(x) \leq \zeta(x)$ implies

$$
\sup _{x \in \bar{T}}\left\{-\zeta^{*}(x)+\log K(m, x)\right\} \geq \sup _{x \in \bar{T}}\{-\zeta(x)+\log K(m, x)\}=r^{*}(m) .
$$

But $-\zeta^{*}(x)+\log K(m, x) \leq r^{*}(m)$ for all $x \in \bar{T}, m \in \bar{D}$ implies

$$
\sup _{x \in \bar{T}}\left\{-\zeta^{*}(x)+\log K(m, x)\right\} \leq r^{*}(m) .
$$

It follows that

$$
r^{*}(m)=\sup _{x \in \bar{T}}\left\{-\zeta^{*}(x)+\log K(m, x)\right\} \quad \text { for } m \in \bar{D} .
$$

Thus $\left(r^{*}, \zeta^{*}\right)=(\log \hat{\rho}, \log \tilde{z})$ for some reflector pair $(\rho, z)$. Note that

$$
\left(r^{*}-r^{*}\left(m^{*}\right), \zeta^{*}+r^{*}\left(m^{*}\right)\right) \in \operatorname{Adm}_{\mathrm{refl}}(\bar{D}, \bar{T}),
$$

where the left hand pair denotes functions shifted by the constants $\pm r^{*}\left(m^{*}\right)$. Using the above, we now have

$$
\begin{aligned}
\mathcal{F}\left(r^{*}-r^{*}\left(m^{*}\right), \zeta^{*}+r^{*}\left(m^{*}\right)\right) & =\mathcal{F}\left(r^{*}, \zeta^{*}\right)=\int_{\bar{D}} r^{*}(m) I(m) d \sigma+\int_{\bar{T}} \zeta^{*}(x) L(x) d x \\
& \leq \int_{\bar{D}} r(m) I(m) d \sigma+\int_{\bar{T}} \zeta(x) L(x) d x=\mathcal{F}(r, \zeta) .
\end{aligned}
$$

This shows that indeed the minimum of $\mathcal{F}$ on $\operatorname{Adm}(\bar{D}, \bar{T})$ is attained on $\operatorname{Adm}_{\text {refl }}(\bar{D}, \bar{T})$. The corollary is an immediate consequence of Theorem 5.3.

\section{A uniqueness result and connection to an optimal transportation problem}

In this section, we show the connection to an optimal transportation problem. This is again quite analogous to the reflector design problems in [5] and [4]. This connection allows us to formulate a uniqueness result for the Reflector Problem I.

For the formulation of the problem, we need the concept of a plan in this context:

Definition 7.1. A plan is a map $P: \bar{D} \rightarrow \bar{T}$ that is measure preserving, that is, we have

$$
\int_{\bar{D}} h(P(m)) I(m) d \sigma=\int_{\bar{T}} h(x) L(x) d x
$$

for any function $h \in C(\bar{T})$. 
A plan $P$ needs only be defined almost everywhere on $\bar{D}$.

The optimal transportation problem associated with intensities $I(m)$ and $L(x)$ satisfying energy conservation $(21)$ and with cost function $\log K(m, x)$ is the following:

Problem III. Maximize the transportation cost

$$
P \mapsto C(P)=\int_{\bar{D}} \log K(m, P(m)) I(m) d \sigma
$$

among all plans $P$.

This problem is again solved by the reflector map of a solution to the Reflector Problem I. In fact, we have the following theorem:

Theorem 7.2. Let $(z, \rho)$ be a solution to the Reflector Problem I. Then the corresponding reflector map $\gamma$ is a plan, and it maximizes the transportation cost (22) among all plans. Any other cost maximizing plan is equal to $\gamma$ almost everywhere on $\operatorname{supp} I \backslash\{m \in \bar{D} \mid I(m)=0\}$.

Proof. Let $(z, \rho)$ be a solution to the Reflector Problem I with corresponding reflector map $\gamma$. By Corollary 5.1, $\gamma$ is in fact a plan. Now let $P$ be another plan. Then

$$
\log \hat{\rho}(m)+\log \tilde{z}(P(m)) \geq \log K(m, P(m))
$$

for almost all $m \in \bar{D}$, and equality holds iff $P(m)=\gamma(m)$. Thus

$$
\begin{aligned}
C(P) & =\int_{\bar{D}} \log K(m, P(m)) I(m) d \sigma \\
& \leq \int_{\bar{D}} \log \hat{\rho}(m) I(m) d \sigma+\int_{\bar{D}} \log \tilde{z}(P(m)) I(m) d \sigma \\
& =\int_{\bar{D}} \log \hat{\rho}(m) I(m) d \sigma+\int_{\bar{T}} \log \tilde{z}(x) L(x) d x \\
& =\int_{\bar{D}} \log \hat{\rho}(m) I(m) d \sigma+\int_{\bar{D}} \log \tilde{z}(\gamma(m)) I(m) d \sigma \\
& =\int_{\bar{D}} \log K(m, \gamma(m))=C(\gamma) .
\end{aligned}
$$

Thus $\gamma$ indeed maximizes the transportation cost among all plans. Moreover, if equality holds in the above estimate, then $\gamma(m)=P(m)$ or $I(m)=0$ for almost all $m \in \bar{D}$. 
We can now state the following theorem, summarizing the main result on the existence of solutions to the Reflector Problem I with an additional uniqueness result:

Theorem 7.3. (Existence and uniqueness for solutions to the Reflector Problem I) There exist solutions to the Reflector Problem I. If $(\rho, z)$ is a solution, then both $\rho$ and $z$ are Lipschitz continuous. The corresponding reflector map $\gamma: \bar{D} \rightarrow \bar{T}$ is single valued almost everywhere on $\bar{D}$. If $(\rho, z)$ and $\left(\rho^{\prime}, z^{\prime}\right)$ are two solutions with reflector maps $\gamma$ and $\gamma^{\prime}$, respectively, then $\gamma(m)=\gamma^{\prime}(m)$ for almost all $m \in \operatorname{supp} I \backslash\{m \in \bar{D} \mid I(m)=0\}$.

Proof. The existence of solutions to Problem I was already obtained in Corollary 6.5. If now $\gamma$ and $\gamma^{\prime}$ are two reflector maps corresponding to two solutions, then both maximize the transportation cost $C$ by Theorem 7.2, and hence we have $\gamma(x)=\gamma\left(x^{\prime}\right)$ for almost all supp $I \backslash\{m \in \bar{D} \mid I(m)=0\}$.

There is a number of open questions for further investigations. For instance, we have assumed certain constraints on the aperture $\bar{D}$, in particular $(0,0,-1) \notin \bar{D}$. From the physical intuition about the problem, these constraints appear to be unnecessary. It would be interesting to extend the theory to these cases as well. Furthermore, a further exploration of the regularity of solutions $(\rho, z)$ depending on the intensities $I$ and $L$ would be desirable. It is expected that some of the currently rapidly growing research on optimal transportation may carry over here. (See the recent survey [10] and the extremely extensive bibliography cited there.)

Acknowledgement: The author would like to thank V. Oliker for useful discussions.

\section{References}

[1] Y. Brenier. Polar factorization and monotone rearrangement of vectorvalued functions. Comm. in Pure and Applied Math., 44, 1991.

[2] L.A. Caffarelli. Allocation maps with general cost functions. Partial Differential Equations and Applications, 177:29-35, 1996.

[3] W. Gangbo and R. J. McCann. Optimal maps in Monge's mass transport problem. C. R. Acad. Sci. Paris Sér. I Math., 321(12):1653-1658, 1995. 
[4] T. Glimm and V. Oliker. Optical design of single reflector systems and the Monge-Kantorovich mass transfer problem. J. Math. Sci. (N. Y.), 117(3):4096-4108, 2003. Nonlinear problems and function theory.

[5] Tilmann Glimm and Vladimir Oliker. Optical design of two-reflector systems, the Monge-Kantorovich mass transfer problem and Fermat's principle. Indiana Univ. Math. J., 53(5):1255-1277, 2004.

[6] V.I. Oliker. Mathematical aspects of design of beam shaping surfaces in geometrical optics. In Trends in Nonlinear Analysis, ed. by M. Kirkilionis, S. Krömker, R. Rannacher, F. Tomi, pages 191-222. Springer-Verlag, 2002.

[7] V.I. Oliker. On the geometry of convex reflectors. PDE's, Submanifolds and Affine Differential Geometry, Banach Center Publications, 57:155169, 2002.

[8] Vladimir Oliker. A rigorous technique for synthesis of offset three-reflector antennas. In Proceedings of the 1995 Antenna Applications Symposium, Allerton Park, Illinois.

[9] Rolf Schneider. Convex bodies: the Brunn-Minkowski theory, volume 44 of Encyclopedia of Mathematics and its Applications. Cambridge University Press, Cambridge, 1993.

[10] Cédric Villani. Optimal transport: Old and new, volume 338 of Grundlehren der Mathematischen Wissenschaften [Fundamental Principles of Mathematical Sciences]. Springer-Verlag, Berlin, 2009.

[11] Xu-Jia Wang. On the design of a reflector antenna. Inverse Problems, 12(3):351-375, 1996.

[12] Xu-Jia Wang. On the design of a reflector antenna. II. Calc. Var. Partial Differential Equations, 20(3):329-341, 2004. 